\title{
ORIGINAL
}

\section{TENDENCIAS DEL ESTADO NUTRICIONAL DE LA POBLACIÓN ESPAÑOLA: RESULTADOS DEL SISTEMA DE MONITORIZACIÓN NUTRICIONAL DE CATALUÑA (1992-2003)}

\author{
Lluís Serra Majem(1,2,3*), Lourdes Ribas Barba(1), Gemma Salvador Castell(3), Blanca \\ Román Viñas(1), Conxa Castell Abat(3), Carmen Cabezas Peña(3), Mari Cruz Pastor Ferrer(4), \\ Blanca Raidó Quintana(1), Joy Ngo de la Cruz(1), Alicia García Álvarez(1), Jaume Serra \\ Farró(3), Luis Salleras Sanmartí(5), Antoni Plasencia Taradach(3) \\ (1) Centro de Investigación en Nutrición Comunitaria, Parc Científic de la Universitat de Barcelona, Barcelona, España. \\ (2) Departamento de Ciencias Clínicas, Universidad de Las Palmas de Gran Canaria, Las Palmas de Gran Canaria, España. \\ (3) Dirección General de Salud Pública, Departament de Salut, Generalitat de Catalunya, Barcelona, España. \\ (4) Departamento de Bioquímica, Hospital Germans Trias i Pujol, Badalona, España. \\ (5) Departamento de Salud Pública, Universitat de Barcelona, Barcelona, España.
}

\section{RESUMEN}

Fundamento: El objetivo de este estudio es analizar los cambios producidos en los hábitos alimentarios y el estado nutricional de la población catalana, a lo largo de 10 años a partir de la información procedente de las dos Encuestas Nutricionales de Cataluña desarrolladas en 1992-93 y 2002-03.

Método: 2641 indivíduos en 1992-93 y 2060 en 2002-03 de 10 a 75 años que participaron en las encuestas. Se utilizaron dos recordatorios de 24 horas, un cuestionario de frecuencia de consumo y un cuestionario general sobre actividad física, conocimientos y actitudes sobre alimentación y uso de suplementos alimentarios. Se midió el peso $(\mathrm{kg})$, la talla $(\mathrm{cm})$ y el perímetro de la cintura $(\mathrm{PC})(\mathrm{cm})$. Se realizó un análisis bioquímico en una submuestra de la población. Se evaluaron colesterol total, colesterol HDL, colesterol LDL, triglicéridos y $\beta$-caroteno, $\alpha$-tocoferol y retinol.

Resultados: Se observa un descenso en la ingesta de fruta, verdura y patata, carne y pescado, y un aumento en el consumo de derivados lácteos y comida rápida (especialmente entre los jóvenes). Se produce un ascenso en el valor medio de índice de masa corporal (IMC) en hombres y de PC en hombres y mujeres. El valor de IMC desciende entre las mujeres (excepto entre las más jóvenes) La prevalencia de obesidad aumenta en hombres (de 9,9\% a 16,6\%), pero no en mujeres. Desciende la colesterolemia media, a expensas del valor de HDL colesterol. Disminuye el porcentaje de población sedentaria en el tiempo libre.

Conclusiones: Es necesaria una política de nutrición que sea efectiva en la promoción de una alimentación acorde con las recomendaciones nutricionales y la dieta mediterránea.

Palabras clave: Encuesta nutricional, estudio transversal, estado nutricional, adecuacidad nutricional, obesidad, monitorización nutricional, tendencias, España, política nutricional.

Correspondencia:

Lluis Serra Majem

Centro de Investigación en Nutrición Comunitaria

Parc Cientific de Barcelona

Baldiri Reixac, 4. Torre D, 4A1

08028 Barcelona

Correo electrónico: 1serra@dcc.ulpgc.es

\section{ABSTRACT}

Trends in the nutritional status of the Spanish population: Results from the Catalan nutrition monitoring system (1992-2003)

Background: The purpose of this study was to evaluate the changes in the nutricional habits and nutricional status of the Catalan population over 10 years interval of the two Catalan Nutricional Surveys conducted in 1992-93 and 2002-03.

Methodo: 2641 individuals in 1992-93 and 2060 individuals in 2002-03 aged 10 to 75 years participated in the surveys. Two 24 hour recall, a food frequency questionnaire, and a general questionnaire with information on physical activity, knowledge and opinions on nutrition and supplements use were administered. Weight $(\mathrm{Kg})$, height $(\mathrm{cm})$ and waist circumference $(\mathrm{WC})$ $(\mathrm{cm})$ were measured. A subsample of the population underwent a biochemical evaluation. Total cholesterol, HDL cholesterol, LDL cholesterol, tryglicerides and $\beta$-carotene, $\alpha$-tocopherol and retinol were measured.

Results: A decrease in the consumption of fruit, vegetables, potatoes, meat and fish and an increase in the consumption of dairy products and fast food (in young individuals) were reported. An increase in the mean value of Body Mass Index (BMI) was observed among males, and an increase in WC mean value was observed in males and females. BMI value decreased in females (except among the younger ones). The prevalence of obesity increased among males (from 9.9\% to 16.6\%), but not in females. Both total cholesterol and HDL cholesterol decreased. A decrease was observed in the percentage of population with sedentary habits during leisure time.

Conclusions: There is a need for an effective nutrition policy promoting healthy nutrition in accordance with the ongoing dietary guidelines.

Key words: Nutrition survey, Cross-sectional surveys, Nutritional status, Nutrient adequacy, Obesity, Nutritional monitoring, Trends, Spain, Nutrition policy. 


\section{INTRODUCCIÓN}

La evaluación del estado nutricional en una población es una herramienta fundamental en el desarrollo de políticas de salud pública para promover unos hábitos nutricionales saludables acorde con las evidencias deducidas de la investigación epidemiológica. La repetición periódica en el tiempo de dicha evaluación, permite observar la tendencia en los parámetros estudiados, evaluar el cumplimiento de los objetivos nutricionales fijados para la población y también evaluar la eficacia de las políticas alimentarias y de nutrición, y de las campañas de promoción de la salud desarrolladas ${ }^{1-3}$. Es con esta finalidad que el Departament de Salut de la Generalitat de Catalunya incluye dentro de los objetivos marcados en el apartado de "Alimentación y Nutrición” del Plan de Salud la necesidad de realizar periódicamente encuestas alimentarias para evaluar el estado nutricional de la población catalana ${ }^{4-9}$.

Desde el año 1986 en que se llevó a cabo la primera encuesta alimentaria poblacional $^{10}$ seguida de diversas campañas informativas y un extenso programa de formación de profesionales, se han realizado dos encuestas más, con una metodología muy similar, la del año 1992-1993 (ENCAT92) $)^{11-15}$ sobre una muestra representativa de 2.754 personas de 6 a 75 años, en la que se combinaron indicadores de consumo de alimentos, antropométricos y bioquímicos, y la última, realizada en 2002-2003 (ENCAT$02)^{16-22}$ que ha permitido evaluar el estado nutricional actual de la población y las tendencias de los distintos indicadores en los últimos 10 años.

El objetivo de este artículo es presentar un resumen de los aspectos más destacables de estas tendencias de la evolución del estado nutricional de la población catalana 1992-2003.

\section{SUJETOS Y MÉTODOS}

Se analizan los datos procedentes de los estudios nutricionales ENCAT-92 y ENCAT-02. En ambos casos se trata de estudios transversales realizados en muestras representativas de la población catalana, la metodología de los cuales se ha descrito con más extensión en otras publicaciones $^{11-22}$.

Muestras: El universo de los estudios lo constituyeron todos los habitantes de Cataluña de 6 a 75 años en ENCAT-92 y de 10 a 85 años en ENCAT-02, siendo la población origen todos los habitantes residentes y censados en los municipios de Cataluña de esas edades. Las muestras de ambos estudios se obtuvieron mediante técnicas de muestreo estratificado según hábitat y aleatoria por conglomerados, siendo la unidad primaria de muestreo los municipios de Cataluña y la última los individuos censados en estos. La confección de las muestras se llevó a cabo de forma proporcional al número de habitantes y al peso específico de cada municipio en la muestra. Los individuos se seleccionaron mediante un proceso de extracción aleatoria estratificado por edad (4 grupos) y sexo a partir del Registro de Población de Cataluña del IDESCAT.

La tasa de participación en las dos encuestas fue similar 69 y $65 \%$ y las muestras obtenidas fueron representativas de la población catalana correspondiente al momento de realización de las encuestas. Para el estudio de las tendencias 1992-2003 se analizan los datos del grupo de edad de 10 a 75 años, con un total de 4701 individuos (2164 hombres y 2537 mujeres); 2641 (1210 hombres y 1431 mujeres) de ENCAT-92 y 2060 (954 hombres y 1106 mujeres) de ENCAT-02 .

Métodos: las encuestas se obtuvieron mediante dos entrevistas domiciliarias llevadas a cabo por un total de 36 dietistas en 
ENCAT-92 y 22 en ENCAT-02, que habían seguido un riguroso proceso de entrenamiento y selección. En ambos estudios se administró un cuestionario general que comprendía entre otras, variables socioeconómicas (profesión, nivel de estudios, etc.) y preguntas sobre hábitos alimentarios, control de enfermedades crónicas, hábito tabáquico, actividad física, uso de suplementos y conocimientos y opiniones sobre nutrición.

La valoración de la ingesta dietética se realizó mediante la combinación de dos recordatorios de 24 horas y un cuestionario de frecuencia de consumo. En la ENCAT92 los recordatorios de 24 horas se realizaron uno en la estación cálida (mayo-julio) y otro en la estación fría (noviembre-diciembre) con el fin de evitar estimaciones sesgadas por la variabilidad estacional. El trabajo de campo de la ENCAT-02 se llevó a cabo desde marzo de 2002 hasta junio de 2003; durante ese periodo se administró a todos los individuos de la muestra un recordatorio de 24 horas. Un $62 \%$ de la muestra realizó una segunda entrevista entre 8 y 30 días después de la primera, obteniéndose otro recordatorio de 24 horas. En ambos estudios las entrevistas se llevaron a cabo todos los días de la semana. La estimación de la raciones consumidas se realizó mediante las medidas caseras propias del hogar del entrevistado. Las entrevistas a personas discapacitadas o con problemas de memoria, y las de menores de 13 años se realizaron con la ayuda de la persona responsable de su cuidado y alimentación. Los encuestadores se encargaron de la codificación e informatización de las entrevistas.

En la codificación de los alimentos del recordatorio de 24 horas y posterior cálculo de la ingesta de energía y nutrientes se emplearon las tablas de composición españolas del CESNID ${ }^{23}$, utilizándose un total de 635 alimentos. Los recordatorios correspondientes a la encuesta ENCAT-92 fueron recodificados y reanalizados de nuevo con las mismas tablas que en ENCAT-02. En cada encuesta a partir de los datos de ingesta de energía y nutrientes de cada recordatorio se procedió a su ajuste por la variabilidad intraindividual según el método de análisis de la variancia descrito inicialmente por Beaton et $\mathrm{al}^{24-27}$.

La valoración del riesgo de ingestas inadecuadas se ha realizado mediante la comparación de los valores de energía y nutrientes con los de las tablas de ingestas recomendadas para la población española del $\mathrm{CSIC}^{28}$, en las que se ha modificado el valor de las recomendaciones de ingesta de folatos (duplicándose $=400 \mu \mathrm{g}$ ) en el grupo de edad de mujeres de 13 a 49 años.

Ambas encuestas incluían un cuestionario de frecuencia de consumo de alimentos. El de ENCAT-92 era de tipo cualitativo y tenía 77 items alimentarios. El de ENCAT02 era cuantitativo y tenía 80 items alimentarios muy parecidos a los de la anterior encuesta.

Durante la entrevista en ambos estudios se obtuvieron distintas mediciones antropométricas en condiciones estándares (peso, talla, perímetros de cintura y cadera, etc.). El peso se obtuvo mediante báscula de baño electrónica Tefal (+/- 0,1 Kg de precisión), la talla mediante tallímetro de pared portátil enrollable Kave (precisión de +/- 0,1 cm) y los perímetros de cintura y cadera mediante cinta métrica inextensible marca Kawe (precisión de +/- 0,1cm).

La prevalencia de obesidad y sobrepeso se analiza en la población de 18 a 75 años a partir de los valores del índice de masa corporal (IMC) calculado a partir del peso $(\mathrm{kg}) /$ talla $\left(\mathrm{m}^{2}\right)$, según los criterios de la $\mathrm{OMS}^{29}$ : sobrepeso $=$ IMC $\geq 25,0-<30,0$ $\mathrm{kg} / \mathrm{m}^{2}$ y obesidad $=\mathrm{IMC} \geq 30,0 \mathrm{~kg} / \mathrm{m}^{2} ; \mathrm{y}$ también a partir de los valores del perímetro de la cintura, categorizados en nivel 1: hombres cintura $=94,0-101,9 \mathrm{~cm}$; muje- 
res cintura $=80,0-87,9 \mathrm{~cm}$ y nivel $2:$ hombres cintura $\geq 102,0 \mathrm{~cm}$ y mujeres cintura $\geq 88,0 \mathrm{~cm}$.

En un $20 \%$ de las entrevistas se realizó un control telefónico para ratificar y en algunos casos completar la información obtenida por los encuestadores.

\section{Evaluación bioquímica}

Una submuestra de los entrevistados de 18 a 75 años que participaron en ENCAT-92 $(\mathrm{n}=893)$ aceptaron participar en la evaluación bioquímica del estado nutricional ${ }^{13-15}$.

En el análisis de ENCAT-02, la muestra ( $n=429$, de 18 a 74 años) provenía de una submuestra de la muestra del Examen de Salud 2002. Dicho Examen de Salud se realizó, a su vez, en una submuestra de la Encuesta de Salud $2000^{30}$. La Encuesta de Salud es realizada de manera periódica por el Departament de Sanitat i Seguretat Social.

En ambos estudios se analizaron distintos parámetros bioquímicos: lipídicos, hematológicos, vitaminas, minerales, proteínas. Las técnicas de obtención de las muestras y análisis han sido ampliamente descritas en otras publicaciones ${ }^{11,13-16}$. En este artículo se evalúan las tendencias observadas en los parámetros lipídicos (colesterol total, colesterol HDL, colesterol LDL, triglicéridos) y en las concentraciones de $\beta$-caroteno, retinol y $\alpha$ tocoferol. El colesterol sérico y los triglicéridos se analizaron por método enzimático CHOD-PAP y GPO-PAP respectivamente, mediante autoanalizador Hitachi 747 y reactivos de Boehringer-Mannheim. El colesterol HDL se determinó por el método Assmann y cols. El colesterol LDL se calculó mediante la fórmula de Friedewald. El $\beta$ caroteno, el retinol y el $\alpha$-tocoferol se analizaron mediante la técnica de cromatografía líquida de alta resolución con detección ultravioleta.

\section{Análisis}

Se analizan, por grupos de edad y sexo, las tendencias 1992-2003 en el consumo de alimentos, en la ingesta de energía y nutrientes, en la estimación de la prevalencia de obesidad y sobrepeso, en la actividad física y en algunos parámetros bioquímicos.

El análisis estadístico se ha realizado mediante el programa SPSS v12. Para la comparación de medias entre dos grupos se ha utilizado el T-test, para más de dos grupos el análisis de la variancia. Para variables no paramétricas se han utilizado los test de Mann-Whitney y Kruskal-Wallis respectivamente. Para las diferencias entre proporciones se ha utilizado el test de la chi-cuadrado.

\section{RESULTADOS}

En la tabla 1 se presenta el consumo de alimentos para toda la muestra por grupos de edad y sexo correspondiente a la encuesta realizada en 1992-93 y a la actual de 2002-03, y en la tabla 2 la ingesta de energía y nutrientes. Es de destacar la disminución en el consumo de patatas, carne, pescado, vísceras, y fruta y verdura, y el aumento en el consumo de zumos de fruta, leche y derivados, bollería, frutos secos y aceite de oliva, si bien estas variaciones tienen matices según el grupo de edad y el sexo.

En cuanto a las tendencias en la ingesta de energía y nutrientes no se observan cambios relevantes, observándose no obstante una disminución en el consumo de proteínas, colesterol, fibra, hierro, vitamina A, niacina y vitamina B12 y un aumento en el consumo de calcio y de grasas y de ácidos grasos como porcentaje de energía. En general las tendencias únicamente son remarcables para la vitamina A (consecuencia de la disminución en el consumo de vís- 
Tabla 1

Tendencias 1992-2003 en el consumo de alimentos de la población catalana de 10 a 75 años

\begin{tabular}{|c|c|c|c|c|c|c|c|c|c|c|c|c|c|c|c|c|c|c|c|c|c|c|c|c|}
\hline \multicolumn{8}{|c|}{ ENCAT 1992-1993 } & & \multicolumn{16}{|c|}{ ENCAT 2002-2003 } \\
\hline \multicolumn{6}{|c|}{$\begin{array}{c}\text { TOTAL } \\
\text { Grupos de edad en años }\end{array}$} & \multirow{2}{*}{\begin{tabular}{|c} 
Hombres \\
$\begin{array}{c}\text { Total } \\
(\mathrm{n}=1210)\end{array}$
\end{tabular}} & \multirow{2}{*}{\begin{tabular}{|c} 
Mujeres \\
$\begin{array}{c}\text { Total } \\
(\mathrm{n}=1431)\end{array}$
\end{tabular}} & & \multicolumn{12}{|c|}{$\begin{array}{c}\text { TOTAL } \\
\text { Grupos de edad en años }\end{array}$} & \multirow{2}{*}{\multicolumn{2}{|c|}{$\begin{array}{c}\text { Hombres } \\
\begin{array}{c}\text { Total } \\
(\mathrm{n}=954)\end{array}\end{array}$}} & \multirow{2}{*}{\multicolumn{2}{|c|}{$\begin{array}{c}\text { Mujeres } \\
\begin{array}{c}\text { Total } \\
(\mathrm{n}=1106)\end{array}\end{array}$}} \\
\hline $\begin{array}{c}10-17 \\
(\mathrm{n}=315)\end{array}$ & $\begin{array}{c}18-24 \\
(\mathrm{n}=541)\end{array}$ & $\begin{array}{c}25-44 \\
(\mathrm{n}=830)\end{array}$ & $\begin{array}{c}45-64 \\
(\mathrm{n}=691)\end{array}$ & \begin{tabular}{|c|}
$65-75$ \\
$(\mathrm{n}=264)$
\end{tabular} & $\begin{array}{c}\text { Total } \\
(\mathrm{n}=\mathbf{2 6 4 1})\end{array}$ & & & & $\begin{array}{l}10-17 \\
(\mathrm{n}=203)\end{array}$ & & $\begin{aligned} \begin{array}{l}18-24 \\
(\mathrm{n}=309\end{array} & \end{aligned}$ & & $\begin{array}{r}25-44 \\
(\mathrm{n}=70\end{array}$ & & $\begin{array}{r}45-64 \\
(\mathrm{n}=602\end{array}$ & & $\begin{array}{r}65-79 \\
(\mathrm{n}=24\end{array}$ & & $\begin{array}{c}\text { Total } \\
(\mathrm{n}=\mathbf{2 0 6}\end{array}$ & & & & & \\
\hline Media & Media & Media & Media & Media & Media & Media & Media & & Media & $\mathbf{p}$ & Media & $\mathbf{p}$ & Media & $\mathbf{p}$ & Media & $\mathbf{p}$ & Media & $\mathbf{p}$ & Media & $\mathbf{p}$ & Media & & Media & \\
\hline 250,4 & 197,2 & 185,6 & 175,8 & 185,4 & 191,2 & 183,0 & 199,0 & Leche & 251,7 & & 229,1 & $\mathrm{~b}$ & 197,5 & & 199,0 & $\mathrm{c}$ & 203,9 & & 208,8 & $\mathrm{c}$ & 197,7 & $\mathrm{~b}$ & 218,3 & \\
\hline 46,7 & 34,1 & 26,5 & 24,5 & 28,5 & 29,4 & 26,4 & 32,2 & Yogures y leches fermentadas & 66,5 & $\mathrm{cc}$ & 59,0 & $\mathrm{c}$ & 65,7 & $\mathrm{c}$ & 53,9 & $\mathrm{c}$ & 55,7 & $\mathrm{c}$ & 60,2 & $\mathrm{c}$ & 55,6 & $\mathrm{c}$ & 64,1 & \\
\hline 18,6 & 25,3 & 20,0 & 16,8 & 14,5 & 18,9 & 19,5 & 18,3 & Quesos & 26,1 & $\mathrm{~b}$ & 28,7 & $\mathrm{~b}$ & 25,9 & $\mathrm{~b}$ & 19,9 & $\mathrm{a}$ & 16,4 & $\mathrm{a}$ & 23,5 & $\mathrm{c}$ & 24,9 & $\mathrm{c}$ & 22,2 & \\
\hline 24,7 & 20,8 & 16,9 & 11,2 & 7,0 & 15,2 & 18,4 & 12,1 & Otros lácteos & 29,7 & & 23,1 & & 22,9 & $\mathrm{a}$ & 15,8 & & 8,0 & & 19,8 & & 24,5 & $\mathrm{a}$ & 15,6 & \\
\hline 340,5 & 277,5 & 248,9 & 228,3 & 235,4 & 254,6 & 247,4 & 261,6 & Total lácteos & 374,1 & $\mathrm{a}$ & 340,0 & $\mathrm{c}$ & 312,1 & $\mathrm{c}$ & 288,6 & $\mathrm{c}$ & 283,9 & $\mathrm{~b}$ & 312,2 & $\mathrm{c}$ & 302,8 & $\mathrm{c}$ & 320,2 & \\
\hline 184,8 & 169,3 & 137,6 & 120,3 & 113,1 & 138,5 & 169,2 & 108,8 & Cereales & 184,5 & & 161,2 & & 147,3 & $\mathrm{~b}$ & 120,9 & & 113,9 & & 141,4 & & 169,4 & & 117,3 & \\
\hline 58,1 & 50,3 & 36,1 & 21,1 & 20,2 & 33,9 & 37,9 & 30,0 & Bollería & 65,1 & & 51,3 & & 41,7 & & 31,0 & a & 21,3 & & 39,9 & & 44,0 & & 36,4 & \\
\hline 83,8 & 77,4 & 74,2 & 75,2 & 71,3 & 75,5 & 84,6 & 66,7 & Patatas & 73,7 & & 62,0 & a & 59,9 & $\mathrm{c}$ & 61,4 & $\mathrm{~b}$ & 56,9 & $\mathrm{~b}$ & 61,7 & $\mathrm{c}$ & 70,8 & $\mathrm{c}$ & 53,8 & \\
\hline 27,5 & 30,2 & 37,2 & 47,1 & 49,5 & 39,7 & 39,8 & 39,7 & Pescado blanco & 19,5 & & 20,2 & $\mathrm{~b}$ & 29,3 & $\mathrm{~b}$ & 38,3 & $\mathrm{a}$ & 39,2 & & 30,8 & c & 31,6 & $\mathrm{a}$ & 30,1 & \\
\hline 6,4 & 11,7 & 16,8 & 17,3 & 13,6 & 14,7 & 16,9 & 12,5 & Pescado azul & 11,9 & $\mathrm{a}$ & 16,7 & $\mathrm{a}$ & 17,5 & $\mathrm{a}$ & 19,1 & a & 14,1 & & 16,9 & $\mathrm{c}$ & 17,3 & $\mathrm{a}$ & 16,5 & \\
\hline 12,0 & 13,2 & 22,6 & 20,4 & 13,3 & 18,2 & 23,1 & 13,6 & Crustáceos y marisco & 10,8 & & 9,9 & $\mathrm{a}$ & 12,7 & $\mathrm{~b}$ & 20,6 & & 12,1 & & 14,3 & & 14,9 & $\mathrm{a}$ & 13,9 & \\
\hline 45,9 & 55,1 & 76,6 & 84,8 & 76,4 & 72,6 & 79,8 & 65,8 & Total pescado & 42,2 & & 46,8 & & 59,4 & $\mathrm{a}$ & 78,0 & & 65,4 & & 62,0 & & 63,7 & & 60,5 & \\
\hline 91,7 & 99,3 & 90,7 & 75,9 & 55,1 & 82,6 & 94,8 & 70,9 & Carnes & 90,8 & & 91,9 & & 76,8 & $\mathrm{c}$ & 61,8 & $\mathrm{c}$ & 47,8 & $\mathrm{a}$ & 72,6 & $\mathrm{c}$ & 84,6 & $\mathrm{c}$ & 62,3 & \\
\hline 51,2 & 45,2 & 35,9 & 24,7 & 21,0 & 33,5 & 41,0 & 26,3 & Embutidos & 43,8 & & 39,4 & $\mathrm{a}$ & 35,8 & & 27,4 & & 19,7 & & 32,8 & & 40,2 & & 26,4 & \\
\hline 1,9 & 1,8 & 2,6 & 3,5 & 4,5 & 2,9 & 3,5 & 2,4 & Vísceras & 0,1 & $\mathrm{a}$ & 0,6 & $\mathrm{a}$ & 1,5 & $\mathrm{a}$ & 2,3 & & 1,9 & & 1,5 & $\mathrm{c}$ & 2,3 & & $\begin{array}{ll}0,9 & \end{array}$ & \\
\hline 52,4 & 54,6 & 54,2 & 52,2 & 55,7 & 53,8 & 53,4 & 54,2 & Aves y caza & 40,8 & & 33,8 & $\mathrm{c}$ & 48,7 & & 45,0 & & 35,8 & $\mathrm{~b}$ & 43,1 & $\mathrm{c}$ & 46,9 & & 39,7 & \\
\hline 25,9 & 27,5 & 29,3 & 24,8 & 17,5 & 25,7 & 29,2 & 22,3 & Huevos & 22,2 & $\mathrm{a}$ & 26,7 & $\mathrm{a}$ & 25,0 & $\mathrm{c}$ & 25,8 & & 20,0 & & 24,6 & $\mathrm{c}$ & 28,1 & $\mathrm{~b}$ & 21,6 & \\
\hline 13,5 & 14,2 & 15,3 & 14,9 & 14,0 & 14,6 & 16,4 & 12,9 & Legumbres & 14,1 & & 11,8 & & 13,7 & $\mathrm{~b}$ & 17,3 & & 16,7 & & 14,9 & b & 15,9 & $\mathrm{a}$ & 13,9 & \\
\hline 3,6 & 2,4 & 4,5 & 3,2 & 2,1 & 3,4 & 4,3 & 2,5 & Frutos secos & 2,6 & & 3,4 & & 4,6 & & 4,3 & $\mathrm{c}$ & 3,3 & $\mathrm{a}$ & 4,0 & c & 4,4 & $\mathrm{c}$ & 3,6 & \\
\hline 194,6 & 236,6 & 270,0 & 372,1 & 375,4 & 301,0 & 287,4 & 314,0 & Frutas & 141,3 & $\mathrm{~b}$ & 141,6 & $\mathrm{c}$ & 193,9 & $\mathrm{c}$ & 293,9 & $\mathrm{c}$ & 313,9 & $\mathrm{~b}$ & 224,3 & $\mathrm{c}$ & 217,6 & $\mathrm{c}$ & 230,0 & \\
\hline 128,1 & 164,0 & 208,4 & 225,9 & 219,3 & 200,4 & 193,9 & 206,6 & Verduras y hortalizas & 121,8 & & 149,7 & $\mathrm{a}$ & 180,8 & $\mathrm{c}$ & 222,4 & & 186,7 & c & 183,2 & $\mathrm{~b}$ & 175,2 & $\mathrm{a}$ & 190,1 & \\
\hline 27,2 & 29,8 & 32,4 & 30,3 & 26,3 & 30,0 & 33,0 & 27,2 & Aceite de oliva & 28,9 & & 29,9 & & 30,5 & & 31,0 & c & 27,2 & & 30,0 & $\mathrm{c}$ & 32,4 & & 27,9 & \\
\hline 39,2 & 41,6 & 43,7 & 39,7 & 33,4 & 40,3 & 44,7 & 36,1 & Total aceites y grasas & 37,4 & & 38,3 & & 38,1 & & 38,0 & $\mathrm{a}$ & 33,3 & & 37,4 & & 41,1 & & 34,3 & \\
\hline 25,3 & 20,1 & 18,3 & 14,4 & 14,3 & 17,6 & 19,7 & 15,6 & Azúcar y dulces & 21,8 & $\mathrm{a}$ & 20,2 & & 17,9 & & 13,7 & & 10,5 & $\mathrm{a}$ & 16,5 & b & 17,9 & $\mathrm{a}$ & 15,3 & \\
\hline 646,7 & 709,9 & 567,5 & 533,4 & 450,4 & 568,6 & 556,4 & 580,4 & Aguas & 831,8 & $\mathrm{c}$ & 795,8 & $\mathrm{c}$ & 795,1 & $\mathrm{c}$ & 690,0 & $\mathrm{c}$ & 602,4 & $\mathrm{c}$ & 745,3 & $\mathrm{c}$ & 762,5 & $\mathrm{c}$ & 730,5 & \\
\hline 9,8 & 56,7 & 90,3 & 81,2 & 60,8 & 70,5 & 68,5 & 72,4 & Cafés y tes & 6,7 & $\mathrm{~b}$ & 52,8 & $\mathrm{a}$ & 85,8 & $\mathrm{c}$ & 95,8 & $\mathrm{a}$ & 64,9 & & 73,5 & & 69,5 & & 77,0 & \\
\hline 104,3 & 120,5 & 81,5 & 38,1 & 23,1 & 68,8 & 88,2 & 50,1 & Bebidas refrescantes & 117,9 & & 147,2 & & 129,0 & $\mathrm{c}$ & 38,1 & & 18,5 & & 91,0 & & 119,7 & & 66,3 & \\
\hline 51,9 & 41,1 & 50,7 & 72,4 & 82,8 & 60,2 & 61,8 & 58,6 & Otras bebidas & 52,8 & & 43,3 & & 53,2 & $\mathrm{~b}$ & 60,1 & $\mathrm{c}$ & 64,9 & $\mathrm{a}$ & 55,1 & $\mathrm{c}$ & 54,7 & $\mathrm{c}$ & 55,5 & \\
\hline 27,4 & 39,0 & 17,4 & 10,0 & 5,5 & 17,7 & 20,0 & 15,4 & Zumos y nectares comerciales & 59,5 & $\mathrm{~b}$ & 49,1 & & 37,9 & $\mathrm{c}$ & 20,0 & $\mathrm{a}$ & 13,6 & & 33,6 & $\mathrm{c}$ & 38,6 & $\mathrm{c}$ & 29,3 & \\
\hline 840,1 & 967,3 & 807,3 & 735,1 & 622,5 & 785,7 & 794,9 & 776,8 & Total bebidas no alcohólicas & 1068,8 & $\mathrm{c}$ & 1088,2 & $\mathrm{c}$ & 1101,1 & $\mathrm{c}$ & 904,1 & $\mathrm{c}$ & 764,3 & $\mathrm{c}$ & 998,5 & $\mathrm{c}$ & 1044,9 & $\mathrm{c}$ & 958,5 & \\
\hline 2,2 & 91,0 & 159,1 & 107,6 & 67,2 & 105,9 & 175,3 & 39,1 & Bebidas alcohólicas & 3,9 & & 50,5 & $\mathrm{a}$ & 94,3 & $\mathrm{c}$ & 123,2 & & 88,9 & & 86,6 & & 135,2 & $\mathrm{~b}$ & 44,7 & \\
\hline
\end{tabular}


Tabla 2

Tendencias 1992-2003 en la ingesta de energía y nutrientes en la población catalana de 10 a 75 años

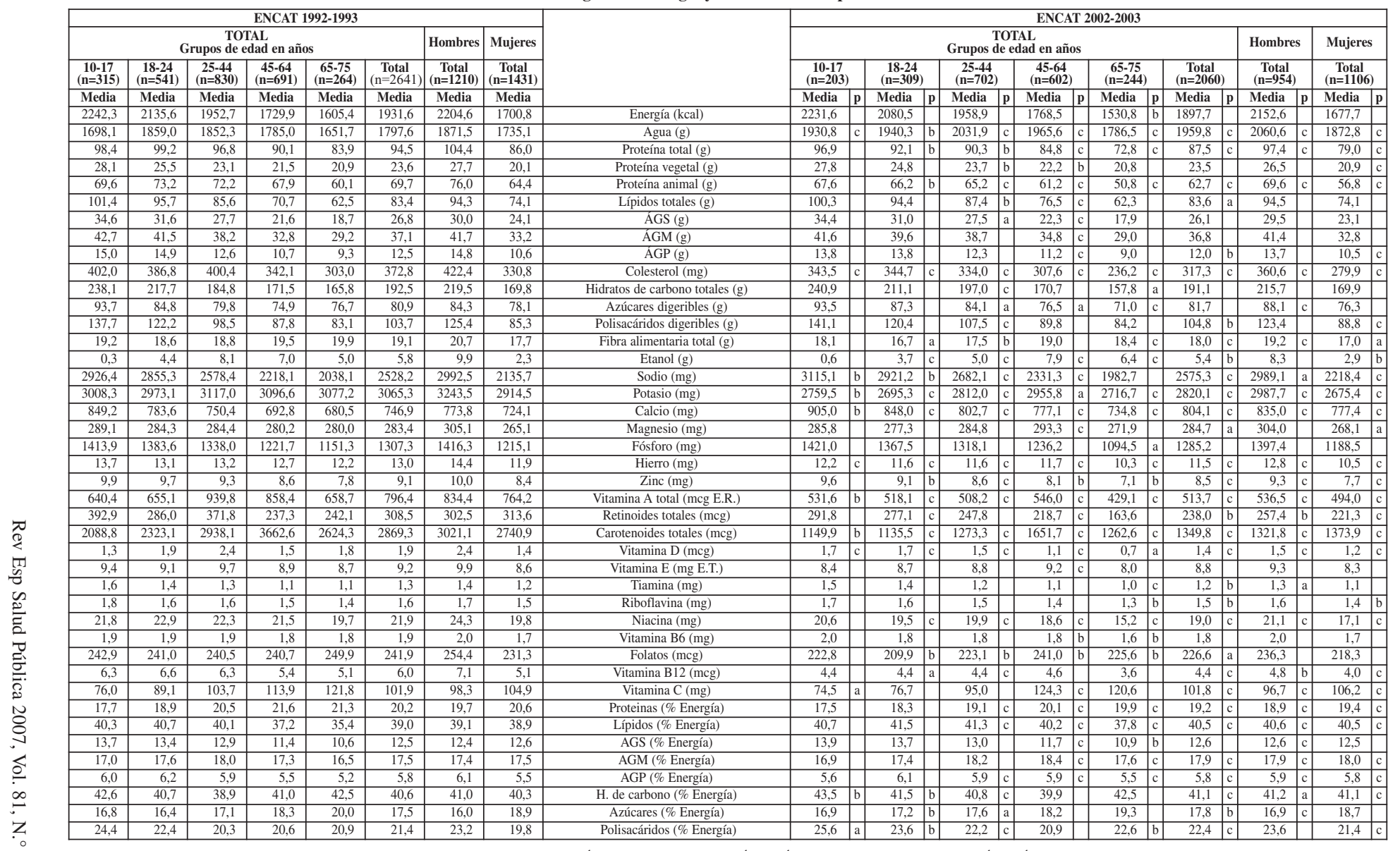

Comparación de medias 1992-2003. significación a = <0,05; b <0,01; c < 001. AGM: Ácidos Grasos Saturados; ÁGM: Ácidos Grasos Monoinsaturados; ÁGP: Ácidos Grasos Polinsaturados. 
Tabla 3

Tendencias 1992-2003 del riego de ingestas inadecuadas de energía y nutrientes en la población catalana de 10 a 75 años

\begin{tabular}{|c|c|c|c|c|c|c|c|c|c|c|c|c|c|c|c|}
\hline & \multicolumn{5}{|c|}{ ENCAT 1992-93 } & \multicolumn{5}{|c|}{ ENCAT 2002-03 } & \multicolumn{5}{|c|}{$\begin{array}{c}\text { Diferencia } \\
\text { (ENCAT 2002-03 -ENCAT 1992-93) }\end{array}$} \\
\hline & \multicolumn{4}{|c|}{ \% de población con ingestas } & \multirow{2}{*}{$\begin{array}{c}\text { Media } \\
\% \text { de } \\
\text { IDR }\end{array}$} & \multicolumn{4}{|c|}{ \% población con ingestas } & \multirow{2}{*}{$\begin{array}{c}\text { Media } \\
\% \text { de } \\
\text { IDR }\end{array}$} & \multicolumn{4}{|c|}{ \% de población con ingestas } & \multirow{2}{*}{$\begin{array}{c}\text { Media } \\
\% \text { de } \\
\text { IDR }\end{array}$} \\
\hline & $\begin{array}{l}<1 / 3 \\
\text { IDR }\end{array}$ & $\begin{array}{l}<1 / 2 \\
\text { IDR }\end{array}$ & $\begin{array}{l}<2 / 3 \\
\text { IDR }\end{array}$ & $<$ IDR & & $\begin{array}{l}<1 / 3 \\
\text { IDR }\end{array}$ & $\begin{array}{l}<1 / 2 \\
\text { IDR }\end{array}$ & $\begin{array}{l}<2 / 3 \\
\text { IDR }\end{array}$ & $<$ IDR & & $\begin{array}{l}<1 / 3 \\
\text { IDR }\end{array}$ & $\begin{array}{l}<1 / 2 \\
\text { IDR }\end{array}$ & $\begin{array}{l}<2 / 3 \\
\text { IDR }\end{array}$ & $<$ IDR & \\
\hline Energía & 0,0 & 2,8 & 22,9 & 88,8 & 79,4 & 0,2 & 1,7 & 22,5 & 93,3 & 77,4 & 0,2 & $-1,1$ & $-0,4$ & 4,5 & $-1,9$ \\
\hline Proteinas & 0,0 & 0,1 & 0,2 & 1,9 & 202,1 & 0,0 & 0,0 & 0,0 & 0,3 & 186,8 & 0,0 & $-0,1$ & $-0,2$ & $-1,6$ & $-15,4$ \\
\hline Calcio & 0,2 & 4,8 & 19,7 & 69,7 & 90,1 & 0,0 & 0,5 & 5,0 & 59,0 & 98,1 & $-0,2$ & $-4,3$ & $-14,7$ & $-10,7$ & 8,0 \\
\hline Hierro & 1,5 & 7,6 & 22,2 & 50,5 & 106,8 & 0,1 & 3,7 & 28,8 & 48,3 & 98,0 & $-1,4$ & $-3,9$ & 6,6 & $-2,2$ & $-8,8$ \\
\hline Zinc & 5,7 & 30,2 & 66,0 & 95,9 & 60,9 & 0,4 & 28,0 & 84,1 & 99,9 & 56,8 & $-5,3$ & $-2,2$ & 18,1 & 4,0 & $-4,2$ \\
\hline Magnesio & 0,3 & 3,9 & 18,8 & 80,3 & 84,3 & 0,0 & 0,6 & 9,6 & 85,9 & 85,3 & $-0,3$ & $-3,3$ & $-9,2$ & 5,6 & 1,0 \\
\hline Tiamina & 0,3 & 1,1 & 4,3 & 24,1 & 134,3 & 0,0 & 0,1 & 0,5 & 10,9 & 124,6 & $-0,3$ & $-1,0$ & $-3,8$ & $-13,2$ & $-9,7$ \\
\hline Riboflavina & 0,2 & 1,3 & 7,6 & 42,9 & 110,4 & 0,1 & 0,2 & 3,1 & 46,8 & 103,0 & $-0,1$ & $-1,1$ & $-4,5$ & 3,9 & $-7,5$ \\
\hline Niacina & 0,2 & 1,0 & 3,6 & 19,4 & 138,0 & 0,0 & 0,0 & 0,2 & 13,5 & 118,1 & $-0,2$ & $-1,0$ & $-3,4$ & $-5,9$ & $-20,0$ \\
\hline Vitamina B6 & 0,3 & 2,0 & 7,8 & 42,1 & 108,9 & 0,0 & 0,1 & 0,9 & 36,3 & 107,3 & $-0,3$ & $-1,9$ & $-6,9$ & $-5,8$ & $-1,6$ \\
\hline Folatos & 4,3 & 15,5 & 29,8 & 52,7 & 125,4 & 0,7 & 13,7 & 29,3 & 45,2 & 99,8 & $-3,6$ & $-1,8$ & $-0,5$ & $-7,5$ & $-25,6$ \\
\hline Vitamina B12 & 0,3 & 0,8 & 2,3 & 6,4 & 300,0 & 0,1 & 0,2 & 0,2 & 0,3 & 220,5 & $-0,2$ & $-0,6$ & $-2,1$ & $-6,1$ & $-79,5$ \\
\hline Vitamina C & 2,8 & 7,3 & 12,7 & 28,0 & 169,8 & 0,0 & 0,1 & 1,4 & 13,4 & 169,8 & $-2,8$ & $-7,2$ & $-11,3$ & $-14,6$ & 0,0 \\
\hline Vitamina A & 9,3 & 20,3 & 42,4 & 100,0 & 90,0 & 1,2 & 26,8 & 80,6 & 99,3 & 57,7 & $-8,1$ & 6,5 & 38,2 & $-0,7$ & $-32,3$ \\
\hline Vitamina D & 78,5 & 89,1 & 91,6 & 93,1 & 37,3 & 73,8 & 92,6 & 97,7 & 100,0 & 27,4 & $-4,7$ & 3,5 & 6,1 & 6,9 & $-9,9$ \\
\hline Vitamina E & 6,5 & 21,9 & 44,0 & 78,7 & 77,8 & 0,6 & 5,7 & 32,8 & 94,3 & 74,2 & $-5,9$ & $-16,2$ & $-11,2$ & 15,6 & $-3,6$ \\
\hline
\end{tabular}

Tabla 4

Tendencias 1992-2003 en las prevalencias de sobrepeso y obesidad

\begin{tabular}{|c|c|c|c|c|c|c|c|c|c|c|c|c|c|c|c|c|}
\hline \multirow{3}{*}{$\begin{array}{c}\text { Grupos } \\
\text { edad (años) }\end{array}$} & \multicolumn{4}{|c|}{ IMC sobrepeso* } & \multicolumn{4}{|c|}{ IMC obesidad** } & \multicolumn{4}{|c|}{ PC nivel $1 * * * *$} & \multicolumn{4}{|c|}{ PC nivel $2^{* * * * *}$} \\
\hline & \multicolumn{2}{|c|}{ Hombres } & \multicolumn{2}{|c|}{ Mujeres } & \multicolumn{2}{|c|}{ Hombres } & \multicolumn{2}{|c|}{ Mujeres } & \multicolumn{2}{|c|}{ Hombres } & \multicolumn{2}{|c|}{ Mujeres } & \multicolumn{2}{|c|}{ Hombres } & \multicolumn{2}{|c|}{ Mujeres } \\
\hline & $\begin{array}{c}1992-3 \\
\%\end{array}$ & $\begin{array}{c}2002-3 \\
\%\end{array}$ & $\begin{array}{c}1992-3 \\
\%\end{array}$ & $\begin{array}{c}2002-3 \\
\%\end{array}$ & $\begin{array}{c}1992-3 \\
\%\end{array}$ & $\begin{array}{c}2002-3 \\
\%\end{array}$ & $\begin{array}{c}1992-3 \\
\%\end{array}$ & $\begin{array}{c}2002-3 \\
\%\end{array}$ & $\begin{array}{c}1992-3 \\
\%\end{array}$ & $\begin{array}{c}2002-3 \\
\%\end{array}$ & $\begin{array}{c}1992-3 \\
\%\end{array}$ & $\begin{array}{c}2002-3 \\
\%\end{array}$ & $\begin{array}{c}1992-3 \\
\%\end{array}$ & $\begin{array}{c}2002-3 \\
\%\end{array}$ & $\begin{array}{c}1992-3 \\
\%\end{array}$ & $\begin{array}{c}2002-3 \\
\%\end{array}$ \\
\hline Total & 44,1 & 43,7 & 29,1 & 30,1 & 9,9 & 16,6 & 15,0 & 15,2 & 21,7 & 23,8 & 21,8 & 17,7 & 13,1 & 24,4 & 24,5 & 31,1 \\
\hline p & \multicolumn{2}{|c|}{$<0,05$} & \multicolumn{2}{|c|}{$n s$} & \multicolumn{2}{|c|}{$<0,05$} & \multicolumn{2}{|c|}{$n s$} & \multicolumn{2}{|c|}{$<0,05$} & \multicolumn{2}{|c|}{$<0,05$} & \multicolumn{2}{|c|}{$<0,05$} & \multicolumn{2}{|c|}{$<0,05$} \\
\hline $18-24$ & 27,0 & 19,8 & 7,3 & 18,1 & 2,5 & 6,9 & 1,7 & 1,3 & 7,7 & 7,8 & 7,3 & 11,9 & 1,3 & 6,0 & 1,7 & 2,5 \\
\hline $25-44$ & 43,2 & 41,7 & 26,7 & 22,9 & 7,0 & 13,3 & 6,6 & 8,7 & 18,8 & 20,3 & 19,3 & 15,5 & 6,2 & 16,3 & 14,0 & 20,7 \\
\hline $45-64$ & 56,4 & 60,0 & 44,7 & 37,5 & 14,7 & 18,4 & 27,8 & 23,8 & 30,9 & 31,2 & 35,8 & 22,4 & 24,8 & 31,2 & 39,2 & 43,4 \\
\hline $65-75$ & 49,5 & 37,9 & 41,8 & 49,1 & 21,5 & 31,0 & 35,6 & 31,3 & 35,5 & 33,0 & 23,6 & 20,0 & 28,0 & 49,6 & 65,3 & 70,9 \\
\hline$p$ & \multicolumn{2}{|c|}{$<0,05$} & \multicolumn{2}{|c|}{$n s$} & \multicolumn{2}{|c|}{$n s$} & \multicolumn{2}{|c|}{$n s$} & \multicolumn{2}{|c|}{$n s$} & \multicolumn{2}{|c|}{$n s$} & \multicolumn{2}{|c|}{$<0,05$} & \\
\hline
\end{tabular}

* Sobrepeso: IMC $\geq 25-<30 \mathrm{~kg} / \mathrm{m}^{2}$. ** Obesidad IMC $>$ ó $=30 \mathrm{~kg} / \mathrm{m}^{2}$. *** Perímetro cintura nivel $1:$ PC $\geq 94-<102 \mathrm{~cm}$ en hombres y $\geq 80-<88 \mathrm{~cm}$ en mujeres. $* * * *$ Perímetro cintura nivel 2 : $\mathrm{PC} \geq 102 \mathrm{~cm}$ en hombres $\mathrm{y} \geq 88 \mathrm{~cm}$ en mujeres.

ceras), vitaminas D y B12 (debidas a la disminución del consumo de carne y pescado) y calcio (consecuencia del incremento en el consumo de derivados lácteos). No se observan cambios en el consumo de calorías ni en hombres ni en mujeres, por tanto los cambios ponderales deberán explicarse sobre todo por cambios en los patrones de actividad física.
En relación a la adecuación nutricional (tabla 3) destaca el incremento del riesgo de ingestas inadecuadas o insuficientes de hierro y vitaminas D y A, y la disminución de la de calcio.

En la tabla 4 se presentan las prevalencias de sobrepeso y obesidad en ambas encuestas por sexos y grupos de edad. Las tendencias 
Tendencias (1992-2003) de los niveles plasmáticos de lípidos y vitaminas de la población catalana de 18 a 74 años

\begin{tabular}{|c|c|c|c|c|c|c|c|c|c|c|c|c|c|c|c|c|c|c|c|c|c|c|}
\hline \multirow{3}{*}{$\begin{array}{l}\text { Grupo de edad (años) } \\
\text { ENCAT }\end{array}$} & \multicolumn{4}{|c|}{$18-34$} & \multicolumn{4}{|c|}{$35-49$} & \multicolumn{4}{|c|}{$50-64$} & \multicolumn{5}{|c|}{$65-74$} & \multicolumn{5}{|c|}{ Total } \\
\hline & \multicolumn{2}{|c|}{1992} & \multicolumn{2}{|c|}{2003} & \multicolumn{2}{|c|}{1992} & \multicolumn{2}{|c|}{2003} & \multicolumn{2}{|c|}{1992} & \multicolumn{2}{|c|}{2003} & \multicolumn{3}{|c|}{1992} & \multicolumn{2}{|c|}{2003} & \multicolumn{2}{|c|}{1992} & \multicolumn{3}{|c|}{2003} \\
\hline & $\mathrm{n}$ & Media & $\mathrm{n}$ & Media $\mathrm{p}$ & $\mathrm{n}$ & Media & $\mathrm{n}$ & Media & $\mathrm{n}$ & Media & $\mathrm{n}$ & Media & & $\mathrm{n}$ & Media & $\mathrm{n}$ & Media $\mathbf{p}$ & $\mathrm{n}$ & Media & $\mathrm{n}$ & Media & \\
\hline Colesterol total (mmol/L) & 266 & 4,64 & 118 & 4,60 & 251 & 5,36 & 115 & 5,14 & 136 & 5,69 & 119 & 5,33 & & 138 & 5,65 & 77 & 5,47 & 891 & 5,27 & 429 & 5,10 & \\
\hline LDL colesterol (mmol/L) & 265 & 2,76 & 117 & 2,82 & 248 & 3,40 & 115 & 3,28 & 232 & 3,66 & 119 & 3,48 & & 136 & 3,57 & 77 & 3,55 & 881 & 3,30 & 428 & 3,26 & \\
\hline HDL colesterol (mmol/L) & 266 & 1,44 & 118 & \begin{tabular}{l|l}
$1,35 a$ \\
$a$
\end{tabular} & 251 & 1,42 & 115 & 1,38 & 237 & 1,45 & 119 & 1,31 & & 138 & 1,47 & 77 & $\begin{array}{l}1,34 \\
\mathrm{a}\end{array}$ & 892 & 1,44 & 429 & 1,34 & \\
\hline Triglicéridos $(\mathrm{mmol} / \mathrm{L})$ & 266 & 0,96 & 118 & 0,93 & 251 & 1,17 & 115 & 1,05 & 236 & 1,29 & 119 & 1,14 & & 138 & 1,39 & 77 & 1,26 & 891 & 1,17 & 429 & 1,08 & \\
\hline$\alpha$-tocoferol $(\mu \mathrm{mol} / \mathrm{L})$ & 112 & 31,32 & 113 & 32,5 & 91 & 31,54 & 112 & 33,00 & 93 & 32,07 & 118 & 34,5 & & 46 & 33,39 & 72 & 34,7 & 342 & 31,88 & 415 & 33,6 & \\
\hline$\beta$-caroteno $(\mu \mathrm{mol} / \mathrm{L})$ & 111 & 0,40 & 109 & 0,40 & 89 & 0,44 & 102 & 0,37 & 92 & 0,43 & 108 & 0,42 & & 45 & 0,45 & 73 & 0,37 & 337 & 0,42 & 392 & 0,39 & \\
\hline Retinol $(\mu \mathrm{mol} / \mathrm{L})$ & 113 & 1,97 & 115 & 2,57 & 91 & 1,82 & 114 & 2,34 & 93 & 1,69 & 119 & 2,29 & & 46 & 1,69 & 76 & $2,19 \mathrm{c}$ & 343 & 1,82 & 424 & 2,36 & \\
\hline
\end{tabular}

${ }^{\mathrm{a}} \mathrm{p}<0,05 ;{ }^{\mathrm{b}} \mathrm{p}<0,01 ;{ }^{\mathrm{c}}<0,001$.

ponderales indican un importante incremento de las prevalencias de obesidad según índice de masa corporal (IMC) en hombres y según el perímetro de cintura, en ambos sexos, mientras que el sobrepeso según IMC solo disminuye en hombres y según PC sólo disminuye en mujeres.

En la tabla 5 se puede observar la evolución de los valores de los niveles plasmáticos de lípidos y vitaminas por grupos de edad en la población catalana. La tendencia del análisis bioquímico muestra que los niveles plasmáticos de colesterol total disminuyen, descenso más evidente entre los hombres que entre las mujeres, especialmente en los grupos de edad de 35 a 64 años. Las cifras de colesterol LDL se mantienen estables en el análisis bioquímico de las dos encuestas analizadas. En cambio, se observa un descenso del nivel sanguíneo del colesterol HDL, de $1,44 \mathrm{mmol} / \mathrm{L}$ en $1992-93$ a $1,34 \mathrm{mmol} / \mathrm{L}$ en 2002-03, cambio que afecta sobre todo a las mujeres. Referente a los niveles de triglicéridos en plasma, se aprecia un descenso de los valores medios. Los niveles de $\alpha$-tocoferol aumentan en todos los grupos de edad analizados. El valor medio de $\beta$-caroteno plasmático disminuye y aumenta el de retinol

\section{DISCUSIÓN}

Las principales causas de mortalidad en los países desarrollados están estrechamen- te relacionadas con la dieta, el consumo de alcohol, el tabaco y una baja actividad física. Por medio de las intervenciones en salud pública se pretende reducir el riesgo medio para la salud de toda la población, así como conseguir un óptimo estado de salud y bienestar. Este objetivo exige la formulación de políticas alimentarias y nutricionales orientadas a la salud de toda la comunidad, eliminando o disminuyendo los factores de riesgo descubiertos ${ }^{31}$.

En España la única encuesta alimentaria a nivel nacional que evalúa el estado nutricional de la población es el Estudio EnKid 1998-2000 ${ }^{32-37}$ realizado en población de 2 a 24 años, no existiendo datos a nivel nacional para la población adulta. A nivel regional varias comunidades autónomas han llevado a cabo estudios nutricionales de su población ${ }^{38}$. La Encuesta Nutricional de Cataluña 2002-03 representa el tercer estudio de nutrición realizado en Cataluña desde 1985 lo que confiere a esta región de un sistema de monitorización nutricional pionero no sólo en España si no también en Europa, permitiendo exponer las tendencias acaecidas en los últimos diez años.

En 1986 se realizó la primera encuesta nutricional en Cataluña ${ }^{10}$. En ella se evidenció un insuficiente consumo de ensaladas y otras verduras, fruta y legumbres en todos los grupos de edad, y un consumo excesivo de alimentos ricos en grasas animales (car- 
nes, embutidos, leche y derivados) y ricos en azúcares (pasteles, dulces, bebidas) sobre todo en los menores de 24 años. Tras esta evaluación inicial se desarrollaron varias campañas nutricionales y un extenso programa de formación a profesionales de la atención primaria de salud, y campañas de sensibilización sobre alimentación equilibrada y salud, programas radiofónicos con intervención de las escuelas; etc. La encuesta de 1992-93 mostró una mejora en los hábitos alimentarios y un acercamiento a los objetivos nutricionales planteados para el año $2000^{6}$ con una disminución calórica, disminución de grasas y aumento de fibra. Después de este estudio siguieron realizándose campañas que fomentaban el seguimiento de la dieta mediterránea, insistiendo en el mantenimiento o aumento (sobre todo en lo más jóvenes) del consumo de frutas y verduras; la utilización del aceite de oliva como grasa de elección, la disminución del consumo de carnes y derivados, el mantenimiento del consumo de pescado y la utilización de lácteos bajos en grasa ${ }^{11}$. Finalmente los resultados mostrados en este estudio destacan las siguientes tendencias alimentarias:

\section{- Aspectos positivos}

1. Aumento del consumo de aceite de oliva, y en particular del aceite de oliva virgen.

2. Aumento del consumo de derivados lácteos, sobre todo bajos en grasa

3. Aumento del consumo de frutos secos.

4. Reducción del consumo de carnes

5. Aumento del consumo de zumos de fruta

- Aspectos negativos

1. Disminución del consumo de frutas y verduras
2. Disminución del consumo de pescado

3. Incremento del consumo de bollería

A pesar de los aspectos positivos destacados en el análisis, se observa aún que la población catalana tiene un patrón alimentario con un escaso seguimiento de las recomendaciones alimentarias de la Sociedad Española de Nutrición Comunitaria ${ }^{39-}$ 41. Es necesario desarrollar una política nutricional efectiva que invierta la tendencia mostrada y que actúe en todos los grupos de población haciendo hincapié en los más jóvenes, no solo por ser los que muestran un mayor desarraigo al patrón de dieta saludable sino también por ser el germen de la población del futuro. En este sentido, el Departament de Salut de la Generalitat de Catalunya ha planificado y desarrollado una Estrategia de Promoción de la Salud intersectorial, denominada PASS (Estrategia de Promoció d' Activitat Física i Alimentació Saludable) en colaboración con otros Departamentos del Gobierno y otras Instituciones y sectores que ya se esta aplicando de forma activa con resultados prometedores ${ }^{42}$.

\section{AGRADECIMIENTOS}

Este estudio ha sido posible gracias a la financiación de la Direcció General de Salut Pública, Departament de Salut de la Generalitat de Catalunya, a través de un acuerdo de colaboración con la Fundación para la Investigación Nutricional.

Los autores quieren expresar su agradecimiento a la Consellera de Salut Marina Geli por su apoyo a las actividades relacionadas con la promoción de la alimentación saludable en Cataluña.

Un agradecimiento especial a todas aquellas personas que fueron entrevistadas y $\sin$ cuya colaboración este trabajo no habría sido posible. 
Grupo de Evaluación y Monitorización del Estado Nutricional de la Población catalana: Lluís Serra Majem, Director (Universidad de Las Palmas de Gran Canaria); Lourdes Ribas Barba, Coordinadora (FIN, Parc Científic de Barcelona); Gemma Salvador (Generalitat de Catalunya); Conxa Castell (Generalitat de Catalunya); Blanca Román Viñas (FIN, Parc Científic de Barcelona ); Jaume Serra (Generalitat de Catalunya); Lluís Jover (Universitat de Barcelona); Ricard Tresserras (Generalitat de Catalunya); Blanca Raidó (FIN, Parc Científic de Barcelona); Andreu Farran (CESNID, Universitat de Barcelona); Joy Ngo (FIN, Parc Científic de Barcelona); Mari Cruz Pastor (Hospital Germans Trias i Pujol, Badalona); Lluís Salleras (Universitat de Barcelona); y Carmen Cabezas, Josep Lluís Taberner, Salvi Juncà, Josep Maria Aragay, Eulàlia Roure Cuspinera, Gonçal Lloveras Vallès († 2003), Antoni Plasencia (Generalitat de Catalunya).

\section{BIBLIOGRAFÍA}

1. World Health Organization. Methodology of nutritional surveillance. Report of a Joint FAO/UNICEF/WHO Expert Committee. World Health Organ Tech Rep Ser. 1976; (593): 1-66.

2. World Health Organization. Global Strategy on Diet, Physical Activity and Health, Resolution of the Fifty-seventh World Health Assembly. WHA57.17. Geneva: WHO; 2004.

3. Lachat C, Van Camp J, De Henauw S, Matthys C, Larondelle Y, Remaut-De Winter AM, et al. A concise overview of national nutrition action plans in the European Union Member States. Public Health Nutr. 2005; 8(3): 266-74.

4. Salleras L, Lloveras G, Serra-Majem L. Nutrition in the health policy context of Catalonia. Eur J Clin Nutr 1993; 47(Suppl 1): s1-3.

5. Generalitat de Catalunya. Departament de Sanitat i Seguretat Social. Document marc per a l'elaboració del Pla de salut de Catalunya. Barcelona: Departament de Sanitat i Seguretat Social; 1991.

6. Generalitat de Catalunya. Departament de Sanitat i Seguretat Social. Pla de salut de Catalunya 1993-
1995. Barcelona: Departament de Sanitat i Seguretat Social; 1994.

7. Generalitat de Catalunya. Departament de Sanitat i Seguretat Social. Pla de salut de Catalunya 19961998. Barcelona: Departament de Sanitat i Seguretat Social; 1997.

8. Generalitat de Catalunya. Departament de Sanitat i Seguretat Social. Pla de salut de Catalunya 19992001. Barcelona: Departament de Sanitat i Seguretat Social; 2001.

9. Generalitat de Catalunya. Departament de Sanitat i Seguretat Social. Estratègies de salut. Pla de salut de Catalunya 2002-2005. Barcelona: Departament de Sanitat i Seguretat Social; 2003.

10. Jiménez A, Cervera P, Sentis J, Canela J, Martínez JM, Lloveras G, et al. Hàbits alimentaris i consum d'aliments a Catalunya. Llibre Blanc. Barcelona: Servei de Promoció de la Salut, Direcció General d'Ordenació i Planificació Sanitària; Generalitat de Catalunya, Departament de Sanitat i Seguretat Social, 1988.

11. Serra Majem L, Ribas Barba L, García Closas R, Ramon JM, Salvador G, Farran A, et al. Llibre Blanc: Avaluació de l'estat nutricional de la població catalana (1992-93). Barcelona: Departament de Sanitat i Seguretat Social, Generalitat de Catalunya; 1996, pp. 1-252.

12. Serra-Majem L, Ribas L, Ramon JM. Compliance with dietary guidelines in the Spanish population. Results from the Catalan Nutrition Survey. Br J Nutr 1999; 81(Suppl 2): s105-12.

13. Garcia Closas R, Serra Majem L, Sabater Sales G, Olmos Castellvell M, Ribas Barba L, Salleras Sanmarti L; Grupo de Investigacion en Nutricion Comunitaria. Distribution of the serum concentration of vitamin C, folic acid and vitamin B12 in a representative sample of the adult population of Catalonia (Spain). Med Clín (Barc). 2002; 118(4): 135-41.

14. Garcia Closas R, Serra Majem L, Chacón P, Olmos M, Ribas L, Salleras L, et al. Distribución de la concentración de lípidos séricos en una muestra representativa de la población adulta de Cataluña. Med Clin (Barc). 1999; 113: 6-12.

15. Garcia Closas R, Serra Majem L, Pastor C, Olmos M, Roman B, Ribas L, et al. Distribución de la concentración sérica de $\beta$-caroteno, retinol y $\alpha$ tocoferol en una muestra representativa de la población adulta de Cataluña. Med Clin (Barc). 2002; 118(7): 256-61. 
16. Serra Majem L, Ribas Barba L, Salvador Castell G, Castell Abat C, Román Viñas B, Serra Farró J, et al. Avaluació de l'estat nutricional de la població catalana 2002-2003. Evolució dels hàbits alimentaris i dels consum d'aliments i nutrients a Catalunya (1992-2003). Barcelona: Departament de Salut, Generalitat de Catalunya; 2006.

17. Ribas-Barba L, Serra-Majem L, Salvador G, Castell C, Cabezas C, Salleras L, et al. Trends in dietary habits and food consumption in Catalonia, Spain (1992-2003). Public Health Nutr. 2007; 10 (11A): 1340-53.

18. Serra-Majem L, Ribas-Barba L, Salvador G, Jover L, Raidó B, Ngo J, et al. Trends in energy and nutrient intake and risk of inadequate intakes in Catalonia, Spain (1992-2003). Public Health Nutr 2007; 10 (11A): 1354-67.

19. García-Álvarez A, Serra-Majem L, Ribas-Barba L, Castell C, Foz M, Uauy R, et al. Obesity and overweight trends in Catalonia, Spain (19922003). Gender and socioeconomic determinants. Public Health Nutr. 2007; 10 (11A): 1368-78.

20. Serra-Majem L, Pastor-Ferrer MC, Castell C, Ribas-Barba L, Román-Viñas B, Font Ribera L, et al. Trends in blood lipids and fat soluble vitamins in Catalonia, Spain (1992-2003). Public Health Nutr. 2007; 10 (11A): 1379-88.

21. Román-Viñas B, Serra-Majem L, Ribas-Barba L, Roure-Cuspinera E, Cabezas C, Vallbona C, et al. Trends in physical activity status in Catalonia, Spain (1992-2003). Public Health Nutr. 2007; 10 (11A): 1389-95.

22. Serra-Majem L, Román-Viñas B, Salvador G, Ribas-Barba L, Ngo J, Castell C, et al. Knowledge, opinions and behaviours related to food and nutrition in Catalonia, Spain (1992-2003). Public Health Nutr. 2007; 10 (11A): 1396-1405.

23. CESNID. Tablas de composición de alimentos. Barcelona, Edicions Universitat de BarcelonaCentre d'Ensenyament Superior de Nutrició i Dietètica; 2002.

24. Beaton GH, Milner J, Corey P, McGuire V, Cousins M, Stewart E, et al. Sources of variance in 24 hour dietary recall data; implications for nutrition study design and interpretation. Am J Clin Nutr. 1979; 32: 2546-59.

25. Beaton GH, Milner J, McGuire V, Feather T, Little JA. Sources of variance in 24 hour dietary recall data: implications for nutrition study design and interpretation. Carbohydrate sources, vitamins and minerals. Am J Clin Nutr. 1983; 37: 986-95.
26. Liu K, Stamler J, Dyer A, McKeever J, McKeever P. Statistical methods to assess and minimize the role of intraindividual variability in obscuring the relationship between dietary lipids and serum cholesterol. J Chronic Dis. 1978; 31: 399-418.

27. Sempos CT, Johnson NE, Smith EL, Gilligan C. Effects of intraindividual and interindividual variation in repeated dietary recalls. Am J Epidemiol. 1985; 121 :120-30.

28. Centro Superior de Investigaciones Científicas (CSIC). Tablas de Ingestas Recomendadas en energía y nutrientes para la población española. Madrid; Instituto de Nutrición, Universidad Complutense de Madrid;1994.

29. World Health Organization. Programme of Nutrition, Family and Reproductive Health. Obesity. Preventing and managing the global epidemic: Report of a WHO consultation on obesity, Geneva: WHO; 1998.

30. Generalitat de Catalunya, Departament de Salut. Examen de salud a la població de Catalunya de 18 a 74 anys. Barcelona: Departament de Salut, 2004. Disponible en: http://www.gencat.net/salut/depsan/units/sanitat/pdf/exsalcat.pdf.

31. Aranceta Bartrina J, Serra Majem L. Objetivos nutricionales y guías dietéticas In: Serra Majem L, Aranceta J, eds. Nutrición y Salud Pública. Métodos, bases científicas y aplicaciones. Barcelona: Masson; 2006.p. 178-83.

32. Serra-Majem L, Ribas L, Ngo J, Ortega RM, García A, Pérez-Rodrigo C, et al. Food, youth and the Mediterranean diet in Spain. Development of KIDMED, Mediterranean Diet Quality Index in children and adolescents.Public Health Nutr. 2004 Oct;7(7):931-5.

33. Serra Majem L, Ribas Barba L, Aranceta Bartrina J, Pérez Rodrigo C, Saavedra Santana P, Peña Quintana L. Childhood and adolescent obesity in Spain. Results of the enKid study (1998-2000). Med Clin (Barc). 2003; 121 (19): 725-32.

34. Pérez-Rodrigo C, Ribas L, Serra-Majem L, Aranceta J.Food preferences of Spanish children and young people: the enKid study. Eur J Clin Nutr. 2003;57 Suppl 1:S45-8.

35. Aranceta J, Pérez-Rodrigo C, Ribas L, SerraMajem L.Sociodemographic and lifestyle determinants of food patterns in Spanish children and adolescents: the enKid study.Eur J Clin Nutr. 2003;57 Suppl 1:S40-4. 
36. Serra-Majem L, Ribas L, Pérez-Rodrigo C, García-Closas R, Peña-Quintana L, Aranceta J.Determinants of nutrient intake among children and adolescents: results from the enKid Study. Ann Nutr Metab. 2002;46 Suppl 1:31-8.

37. Serra-Majem L, García-Closas R, Ribas L, PérezRodrigo C, Aranceta J. Food patterns of Spanish schoolchildren and adolescents: The enKid Study. Public Health Nutr. 2001 Dec;4(6A): 1433-8.

38. Elmadfa I, Weichselbaum E, Konig J, de Winter A-M R, Trolle E, Haapala I, et al. European nutrition and health report 2004. Forum Nutr 2005; (58): 1-220.

39. Serra Majem L, Aranceta J, Mataix J. Documento de consenso: guías alimentarias para la población española. Barcelona: SG-Editores; 1995.
40. Aranceta J, Serra-Majem L on behalf of the Working Party for the Development of Food-Based Dietary Guidelines for the Spanish Population. Dietary guidelines for the Spanish population. Public Health Nutr 2001; 4(6A): 1403-8.

41. Dapcich V, Salvador G, Ribas L, Pérez Rodrigo C, Aranceta J, Serra Majem 1. Guía de la Alimentación saludable. Madrid; SENC-Everest; 2005, pp. 1-107.

42. Serra-Majem L, Ribas-Barba L, Salvador G, Serra J, Castell C, Cabezas C, Plasencia A. Compliance with dietary guidelines in the Catalan population: basis for a nutrition policy at the regional level (the PASS strategy). Public Health Nutr. 2007; 10(11A):1406-14. 